\title{
Radiological progression of penicillin-sensitive Staphylococcus aureus aortitis
}

Hong Kong Med J 2019;25:329.e1-2

S Zheng *

https://doi.org/10.12809/hkmj187616

In June 2017, a 58-year-old man, with no known cardiovascular risk factors, was admitted to a hospital in Singapore, presenting with a 1-week history of fever and progressively worsening epigastric pain On the first day of his symptoms, he had visited the emergency department at another hospital where a computed tomography (CT) scan of the abdomen performed had not revealed significant pathology (Fig 1). He was treated symptomatically as for a viral infection, but he represented to our hospital a week later without significant symptomatic improvement. The patient had a known history of reaction to penicillin and a history of traumatic spinal injury more than 20 years ago requiring spinal instrumentation at the L4/5 level. On examination, the patient was febrile but haemodynamically stable. Abdominal examination revealed epigastric tenderness on deep palpation. Cardiorespiratory examination was unremarkable. The patient had leucocytosis of $15.37 \times 10^{3} / \mathrm{uL}$, raised C-reactive protein level of $109.2 \mathrm{mg} / \mathrm{L}$, and erythrocyte sedimentation rate of $46 \mathrm{~mm} / \mathrm{h}$. Renal and liver function tests, and serum amylase and lipase levels were unremarkable. $\mathrm{He}$ was started empirically on intravenous ceftriaxone following blood cultures.

A new CT scan of the abdomen was performed, revealing an anterior pre- and para-vertebral soft tissue mass with focal hypodensities surrounding the aorta at the T12-L1 level (Fig 2). Blood culture results (which were received 3 days later) were positive for

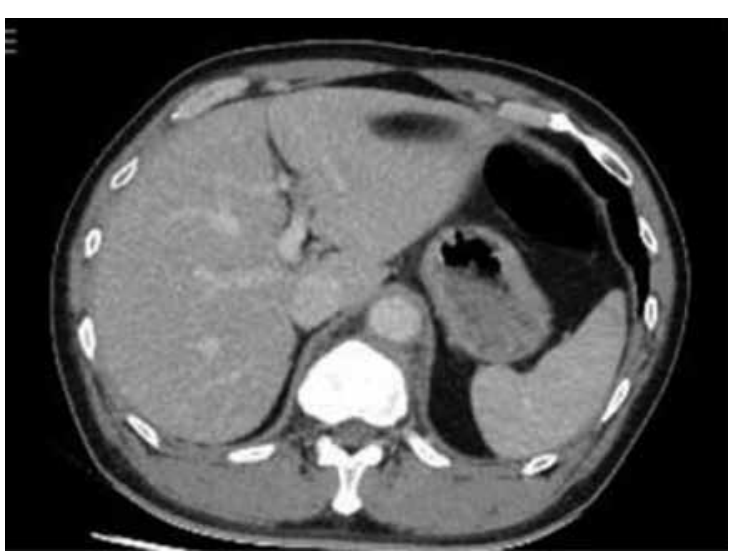

FIG I. Normal computed tomography abdominal image without significant periaortic collection at day I of symptoms penicillin-sensitive Staphylococcus aureus. Magnetic resonance imaging of the thoracolumbar spine illustrated paravertebral soft tissue thickening at level of T12-L1 that appears multiloculated with rim enhancement, suggestive of an underlying paravertebral abscess without epidural extension. A transthoracic echocardiogram did not reveal any valvular lesions. The presence of an inflammatory collection around the aorta prompted concerns for an infectious aortitis.

Antimicrobial therapy was switched to intravenous cefazolin, which was continued for 6 weeks with symptomatic improvement. Repeated blood cultures did not show evidence of persistent $S$ aureus bacteraemia. Monitoring of C-reactive protein level and erythrocyte sedimentation rate showed gradual improvement. A CT aortogram, after 6 weeks of parenteral antibiotics, showed interval improvement of the paravertebral collections and soft tissue thickening around the aorta, suggesting improving aortitis (Fig 3). Antibiotics were switched to oral trimethoprim and sulfamethoxazole. Another CT aortogram 8 months later showed complete resolution of aortitis and paravertebral abscess (Fig 4).

In the antibiotic era, infectious aortitis is a rare clinical entity. Gram-positive micro-organisms are most commonly implicated, in up to $60 \%$ of cases, with $S$ aureus being the most frequently encountered micro-organism. Other micro-organisms commonly

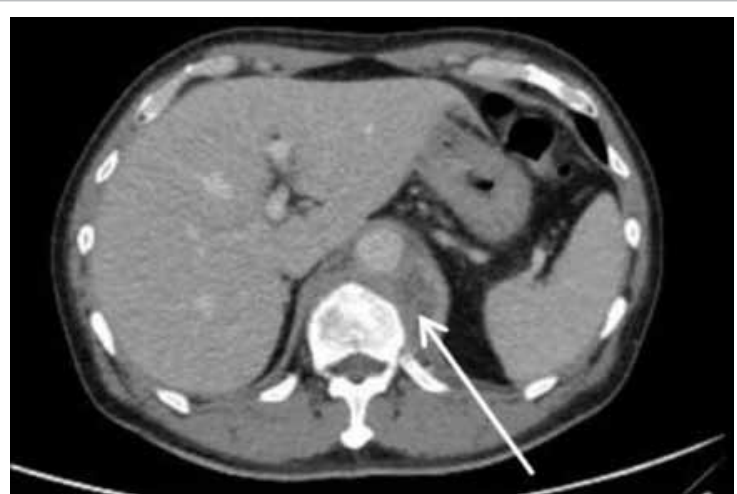

FIG 2. Computed tomography image of the abdomen at I week after symptom onset, revealing an anterior pre- and para-vertebral soft tissue mass with focal hypodensities surrounding the aorta the TI2-LI level 


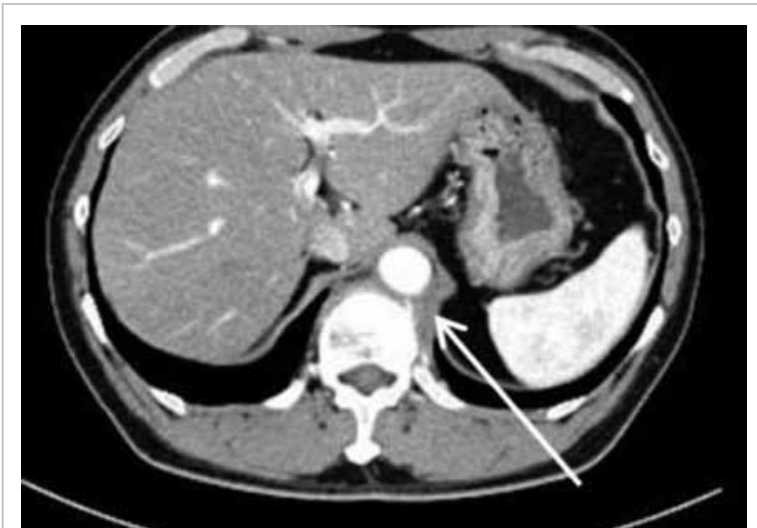

FIG 3. Computed tomography aortogram, after 6 weeks of parental antibiotics, showing interval improvement but residual paravertebral collections and soft tissue thickening around the aorta

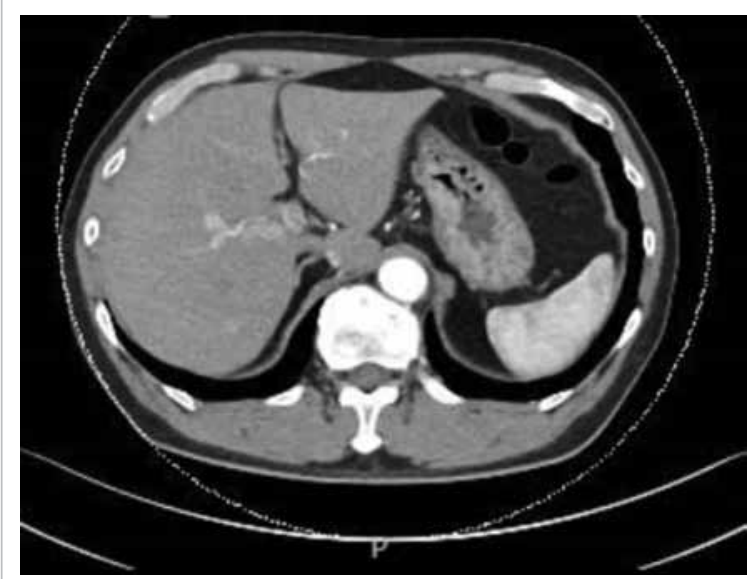

FIG 4. Complete resolution of aortitis on computed tomography aortogram 8 months later

implicated include Enterococcus species, Streptococcus pneumoniae, Salmonella species, Mycobacterium tuberculosis, and in the more distant past, syphilis. ${ }^{1}$ Rare case reports have featured the radiological evolution of infectious aortitis while on conservative treatment, and these often illustrate peri-aortic soft tissue masses progressing to aneurysmal formation from $S$ aureus or Salmonella species infection..$^{1-5}$ Prompt antimicrobial therapy is crucial along with endovascular or surgical intervention. ${ }^{5}$ Our patient demonstrated radiological normality to pathology within a week of symptom onset and subsequent improvement while on conservative therapy alone, followed by full radiological resolution. We believe that his successful recovery is in part due to early appropriate and prolonged antimicrobial therapy.

\section{Author contributions}

The author designed the study, contributed to acquisition and analysis of data, drafted the article, and contributed to the critical revision for important intellectual content. The author had full access to the data, contributed to the study, approved the final version for publication, and takes responsibility for its accuracy and integrity.

\section{Conflicts of interest}

The author has disclosed no conflicts of interest.

\section{Funding/support}

This research received no specific grant from any funding agency in the public, commercial, or not-for-profit sectors.

\section{Ethics approval}

This study was conducted in accordance with the principles outlined in the Declaration of Helsinki.

S Zheng *, MB, BS, MRCP

Department of General Medicine, Sengkang General Hospital, Singapore

* Corresponding author: zheng.shuwei@singhealth.com.sg

\section{References}

1. Cevasco M, Menard MT, Bafford R, McNamee CJ. Acute infectious pseudoaneurysm of the descending thoracic aorta and review of infectious aortitis. Vasc Endovascular Surg 2010;44:697-700.

2. Carreras M, Larena JA, Tabernero G, Langara E, Pena JM. Evolution of salmonella aortitis towards the formation of abdominal aneurysm. Eur Radiol 1997;7:54-6.

3. Rozenblit A, Bennett J, Suggs W. Evolution of the infected abdominal aortic aneurysm: CT observation of early aortitis. Abdom Imaging 1996;21:512-4.

4. Wein M, Bartel T, Kabatnik M, Sadony V, Dirsch Olaf, Erbel R. Rapid progression of bacterial aortitis to an ascending aortic mycotic aneurysm documented by transesophageal echocardiography. J Am Soc Echocardiogr 2001;14:646-9.

5. Kan CD, Lee HL, Yang YJ. Outcome after endovascular stent graft treatment for mycotic aortic aneurysm: a systematic review. J Vasc Surg 2007;46:906-12. 